\title{
UNE CRISE STRUCTURELLE AU BRESIL
}

\section{Pierre Salama}

Universidade Paris XIII

\section{UNE CRISE STRUCTURELLE AU BRESIL}

Résumé: II s'agit de mettre en évidence l'ampleur et la nature de la récente crise structurelle brésilienne, tant au sens économique que politique. La crise politique aggrave la crise économique, et vice et versa, pouvant entrainer une crise institutionnelle. La lutte de classe devient plus forte, tandis que la légitimation étatique chute. Alors, une tentative de coup d'État médiatico-politique est en cours.

Mots-clefs: Crise structurelle brésilienne, lutte de classe, coup d'État.

\section{STRUCTURAL CRISIS IN BRAZIL}

Abstract: It is to highlight the extent and nature of the recent Brazilian structural crisis, both in the economic as the political sense. The political crisis worsens the economic crisis, and vice versa, may causing an institutional crisis. The class struggle becomes more acute, while that state legitimacy weakens. So an attempt to media - legal coup was triggered.

Key words: Brazilian structural crisis, class struggle, State coup. 


\section{INTRODUCTION}

Dans les années 1980, l'Amérique Latine est rattrapée puis dépassée par les dragons asiatiques (Corée du sud, Taïwan, Hong-Kong et Singapour). Cependant, dans les années 1990-2000, l'Amérique latine renoue avec une croissance modérée et le revenu par habitant des principaux pays (Argentine, Brésil, Mexique, Colombie...) s'établit en 2012 à un peu plus du quart du revenu par tête des Etats-Unis alors que ceux de la Corée du sud et de Taïwan se situent autour de $70 \%$ pour le premier et $80 \%$ pour le second de celui des Etats-Unis. Malgré cette croissance plus élevée, l'Amérique latine se marginalise. Même constat si on compare l'Amérique latine avec la Chine. Le revenu par tête de la Chine (14135 dollars PPA en 2014) est très proche de celui du Brésil dès 2014 (15500 dollars PPA en 2014) et le dépasse en 2015, alors qu'il s'élevait à 309, 2 dollars contre 4825,6 dollars au Brésil en 1980.

Au vu de ces données, on peut donc déduire que la croissance est tout compte fait relativement modeste en Amérique latine et au Brésil, pays le plus puissant et le plus peuplé de la région. Et qu'aujourd'hui nombre de pays asiatiques ont un revenu par tête plus élevé que dans les pays émergents latino-américains. Pourtant, dès l'arrivée du Président Lula da Silva au pouvoir, la croissance s'accélère, la pauvreté baisse drastiquement mais contrairement à ce qui se dit officiellement, les riches deviennent plus riches et la part des $1 \%$ dans le revenu des plus riches s'accroit comme dans l'ensemble des pays avancés, le Brésil ne faisant pas exception sauf dans sa capacité à diminuer la pauvreté ${ }^{1}$ (SALAMA, 2014, 2012 apud SALAMA, 2015). Nombre d'économistes s'interrogent alors sur la possibilité que le Brésil devienne un nouvel eldorado. Le mythe s'effondre dès 2011 et les rêves s'envolent, le miracle devient mirage, voire cauchemar dès 2014.

Selon I'Instituto Brasileiro de Geografia e Estatística (IBGE), le taux de croissance du PIB brésilien baisse dès $2011: 3,9 \%$ en $2011,1,9 \%$ en 2012, $3 \%$ en $2013,0,1 \%$ en 2014 . La croissance fléchit avant même que ne s'effondrent les prix des matières premières. En 2015, les prévisions sont continuellement revues à la baisse et l'année se termine avec une chute du PIB de $-3,8 \%$ et le PIB par tête s'effondre: $-4.6 \%$. La consommation des ménages baisse de $3,9 \%$, le taux d'investissement tombe en chute libre: $-14,6 \%$ annonçant ainsi la poursuite de la crise pour 2016, voire 2017, la production baisse fortement: $-6,8 \%$, et seuls le secteur agricole croit légèrement: $1,8 \%$ et les exportations croissent entre février 2015 et février 2016 de 4,6\%. Malgré la chute du cours des matières premières, la contrainte externe s'atténue ${ }^{2}$. Ce qui n'est pas le cas, loin de là de la contrainte interne (solde budgétaire) comme nous le verrons plus loin.
L'ampleur de la crise est telle aujourd'hui que quasiment toutes les couches sociales sont affectées, les revenus du travail baissent, les profits fléchissent y compris les dividendes versés, les acquits sociaux des années 2000 sont rognés et menacés de disparaitre en grande partie. Dans ce contexte où crise se conjugue avec reprise inflationniste, le chômage s'accentue, les salaires baissent, la pauvreté s'accroit de nouveau et ce d'autant plus que la hausse de prix de première nécessité est plus élevée que celle de l'indice général des prix ${ }^{3}$.

La crise économique est également une crise politique pour trois raisons. La première vient du mensonge originel de la campagne électorale. Déniant la crise qui pointait, Dilma Rousseff a construit sa campagne sur une dénonciation virulente du néolibéralisme de son adversaire pour appliquer dès sa nomination pour un second mandat la politique de son adversaire...sans même une parenthèse où elle aurait pu décider quelques mesures correspondant à ses promesses. La seconde est la force de la crise et de ses conséquences sociales. La troisième est la corruption révélée avec force par le scandale de la Pétrobras (dit du lava jato), les poursuites engagées contre plusieurs hauts responsables du Parti des travailleurs, les menaces d'un empeachment engagé contre la présidente de manière pour le moins politicienne de la part de l'opposition. La crédibilité politique, et derrière la légitimité de la présidente nouvellement réélue chute immédiatement et se situe à un niveau extrêmement faible. Cette crise politique provoque un déchirement à l'intérieur du Partido dos Trabalhadores (PT), des difficultés croissantes de maintenir la coalition pour pouvoir gouverner, des majorités introuvables dans les chambres qui rendent peu cohérentes les décisions prises, quelle que soit leur orientation parfois. A un déficit de légitimité s'ajoute ainsi un déficit de rationalité. Au final, les mesures libérales décidées perdent de leur cohérence, aggravant une crise qu'elles engendrent.

Au Brésil, la crise est à la fois une économique et une crise politique, l'une entretenant l'autre, et vice et versa. II s'agit d'une crise structurelle pouvant dégénérer en crise institutionnelle. (SALAMA, 2015).

\section{DIAGNOSTIC, LES OCCASIONS MANQUÉES}

Le vent arrière qui a poussé en avant l'économie brésilienne dans les années 2000 a favorisé 1/ la désindustrialisation, 2/ une insertion dans la division internationale du travail portant sur des produits à intensité technologique de plus en plus faible (à l'exception de quelques secteurs comme l'aéronautique), $3 /$ un déficit de la balance commerciale de produits industriels depuis 2008 qui s'est transformé rapidement en gouffre que parvenait de moins en moins a combler l'excédent provenant 
de la vente de matières premières, 4/ enfin un déphasage dans l'industrie entre la demande et l'offre de main d'œuvre, les demandes de travail qualifié des entreprises baissant relativement alors même que les offres de travail qualifiés augmentaient grâce aux efforts faits en matière d'éducation (allongement du nombre d'années scolaires).

Tableau 1 - Un resserrement des revenus selon le nombre d'années passés à l'école

\begin{tabular}{l|l|l|l|l}
\hline & 1997 & 2002 & 2004 & 2009 \\
\hline (4 à 7$) /(0$ à 3) & 1.46 & 1.47 & 1.46 & 1.31 \\
\hline (8 à 11)/(4 à 7$)$ & 1.64 & 1.50 & 1.43 & 1.38 \\
\hline (12 et plus)/(8 à 11) & 1.73 & 1.85 & 1.74 & 1.54 \\
\hline (15 et plus)/(12 à 14) & 1.89 & 2.07 & 2.21 & 2.28 \\
\hline
\end{tabular}

Source: ROCHA, S. R. Indicateurs et Politique Sociale au Brésil. In: MAISON DES SCIENCES DE L'HOMME, 2011. Anais... Paris, 2011. Power point. À partir des données de l'IBGE et de la PNAD, * homme urbains occupés au moins 20 h par semaine.

De cet effet de ciseau, en période de plein emploi, il en résulté un resserrement des inégalités salariales selon le nombre d'années suivis à l'école (Tableau 1), les jeunes qualifiés à la recherche d'un emploi ne trouvant pas un travail à la hauteur de leur qualification (déclassement et désaffiliation) de nature à provoquer un profond sentiment de ressentiment, resserrement accentué par la hausse très importante du salaire minimum qui a favorisé surtout les salariés non qualifiés ou peu qualifiés (Tableau 2).

La hausse soutenue du salaire minimum, la baisse du chômage dans la première décennie $\mathrm{du} X X I^{\circ}$ siècle, ont participé fortement à la baisse de la pauvreté, ce qui d'un point de vue politique est à mettre à l'actif des gouvernements Lula 1 et 2 et Dilma 1, mais en l'absence d'une politique structurelle visant à stopper la désindustrialisation, se sont traduites par une forte augmentation des importations. Le wage led, au lieu de stimuler le marché interne, a ainsi conduit à un déficit de plus en plus important de la balance commerciale des produits de l'industrie de transformation dès 2008 et à des difficultés croissantes d'exporter des produits industriels plus ou moins sophistiqués (Figure 1) ainsi que le souligne l'ensemble des publications de I'Instituto de Estudos para o Desenvolvimento Industrial (IEDI).
Le coût unitaire du travail ${ }^{4}$ augmente considérablement traduisant une baisse de la compétitivité prononcée (Figure 2), conséquence d'une hausse très faible de la productivité du travail sur la période, d'une appréciation élevée de la monnaie nationale et de la hausse prononcée des salaires réels. II eût été possible de compenser la hausse des salaires réels et d'annuler ses effets négatifs sur la compétitivité $1 /$ si une politique industrielle et de recherche avait été élaborée avec suffisamment d'audace et de moyens pour stimuler la croissance de la productivité du travail et $2 / \mathrm{si}$ une politique de change déprécié avait été menée, stérilisant les apports de devise. Cela n'a pas été fait. Cela se paie aujourd'hui au prix fort, pas pour tous mais pour la grande majorité.

Figure 1 - Industrie de transformation, production selon l'intensité technologique et solde de la balance commerciale de cette industrie 1995-2013

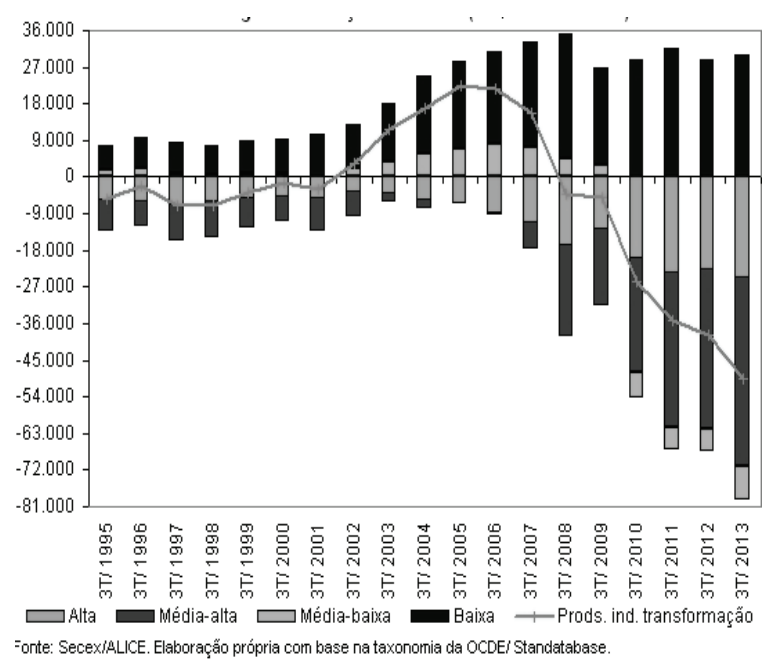

Figure 2 - prix des biens importés et coût unitaire du travail en réal, $2004=100$

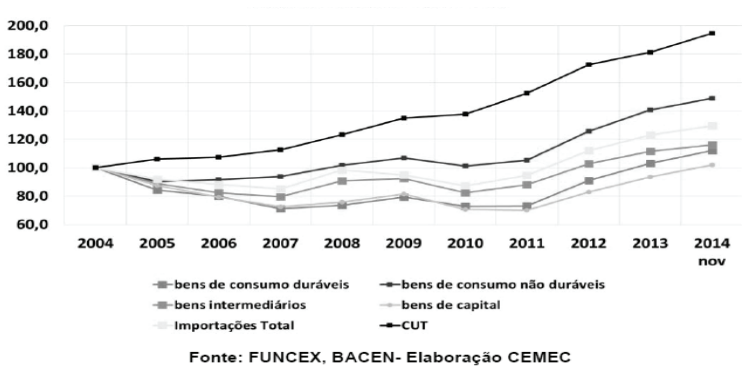

Tableau 2 - Taux de croissance du salaire minimum en termes reels $\mathbf{2 0 0 0 - 2 0 1 5}$

\begin{tabular}{|c|c|c|c|c|c|c|c|c|c|c|c|c|c|c|c|}
\hline 2000 & 2001 & 2002 & 2003 & 2004 & 2005 & 2006 & 2007 & 2008 & 2009 & 2010 & 2011 & 2012 & 2013 & 2014 & 2015 \\
\hline 5.5 & 8.9 & -3.2 & 8,7 & 2.1 & 9.8 & 13.5 & 3.2 & 2.6 & 7.6 & 3 & 0.7 & 7.5 & 3 & 1 & 1 \\
\hline
\end{tabular}


Figure 3 - Rentabilité sur patrimoine net

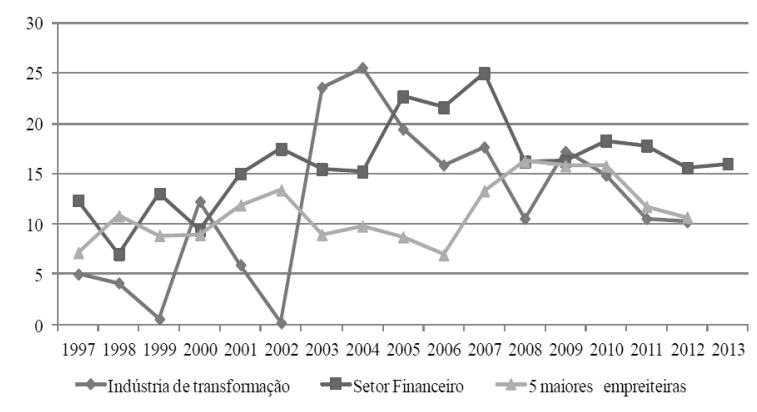

Source: Préparé selon les données CEMEC à partir des informations fournies par la revue Exame pour l'industrie de transformation (sauf des branches pétrole, gaz) et pour le secteur financier à partir de Gazetta Mercantil et O valor.

Cette crise vient donc de loin. On peut le constater en analysant la rentabilité des entreprises (Figure 3), celle-ci baisse bien avant celle du cours des matières premières. C'est pourquoi seules des mesures d'ordre structurelle pourrait la surmonter. Ne pas les prendre aggraverait la crise sur le moyen terme et aurait pour conséquence un cout social très important de nature a effacer les conquêtes sociales. A terme, mais a terme éloigné, une reprise aurait lieu certes mais dans le cadre d'une société davantage exclusive qu'elle ne l'est a présent. Comme souligné précédemment la non résolution de la crise politique ajoute à la confusion des politiques économiques décidées selon l'évolution des rapports de force et rend encore plus difficile la définition d'une politique cohérente quelle qu'elle soit.

De manière générale, aussi paradoxale que cela puisse paraitre, une crise est à la fois nécessaire et dangereuse pour la reproduction du capital. Elle est nécessaire parce que nombre de capitaux ne permettent plus une valorisation satisfaisante compte tenu de la concurrence. Ils doivent donc être détruits (faillites d'entreprises) ou bien dévalorisé. Elle est dangereuse parce que si elle ouvre la possibilité d'accroître le taux d'exploitation (abaisser les salaires, imposer de nouvelles conditions de travail) elle peut entrainer une résistance des salariés et une dynamique de contestation difficilement contrôlable alimentant une remise en cause de la domination aveugle du Capital. Dangereuse enfin parce qu'elle délégitime le plus souvent les gouvernements lorsque ceux-ci optent pour un rétablissement des profits au détriment des salariés.

Il est possible de sortir d'une crise lorsque les conditions d'exploitation sont assainies, mais il est possible aussi de dépasser temporairement et superficiellement une situation de crise de manière superficielle grâce à des politiques de relance du gouvernement qui ne s'attaqueraient pas aux vrais problèmes. Dans ce cas, celle-ci ressurgit, parfois avec d'autant plus de force que les conditions de valorisation n'ont pas été suffisamment rétablies. Mais il est aussi possible de dépasser une crise structurelle en changeant de régime de croissance sans pour autant faire peser sur le travail les sacrifices.

Concrètement, changer de régime de croissance au Brésil aujourd'hui implique d'abandonner le régime de croissance rentier lié à la reprimarisation et à la financiarisation ${ }^{5}$ de l'économie, redistributif vis-à-vis des pauvres, pour un régime de croissance lié à la dynamique du marché intérieur à la condition toutefois que les conditions de compétitivité soit rétablies par des politiques industrielle, de change, et de productivité audacieuses.

La crise actuelle est la conséquence de l'absence de réformes structurelles - non faites lorsqu'il était possible de les mettre en œuvre lors de la phase de bonanza des matières premières comme la réforme fiscale, une politique industrielle pensée.

\section{DÉFICITS DE LÉGITIMITÉ ET DE RATIONALITÉ, À LA RECHERCHE D'UNE ALTERNATIVE}

Les difficultés économiques actuelles manifestent l'épuisement des modèles de croissance des années 2000 et l'impréparation des gouvernements à faire face à des difficultés pourtant prévisibles ${ }^{6}$. Ces difficultés d'ordre structurelles appellent des mesures structurelles. II n'y a pas de sorties techniques à ces difficultés. Face à une crise structurelle, les sorties de crise sont d'ordre à la fois politique et technique. Si ces mesures n'ont pas été prises plus tôt c'est parce qu'elles suscitaient des conflits d'intérêt puissants. La politique vis-à-vis du taux de change avait ses lobbies, ceux d'un refus de procéder à une véritable réforme fiscale avait les siens, plus ou moins les mêmes, etc. La disparition (temporaire) de la contrainte externe (excédents commerciaux massifs mais déclinants dès 2006, entrées importantes de capitaux compensant le déficit croissant de la balance des comptes courants) dans les années 2000 a rendu moins urgent de procéder à ces réformes structurelles, de contourner les contradictions, de rendre possible à la fois la diminution de la pauvreté et la multiplication des milliardaires. Elle se paie aujourd'hui.

Sans un bon diagnostic et des mesures de politiques économiques appropriées, les politiques économiques traditionnelles ont la légitimité de leur fausse évidence. Que faire si le déficit budgétaire est insoutenable - et il l'est devenu - si ce n'est chercher à le réduire et donc couper dans les dépenses? Mais, ce faisant, elles ne font qu'aggraver la crise. C'est ce qui se passe actuellement. La politique d'ajustement fiscal, décidée dès le début du second mandat de Dilma Rousseff, n'est pas de nature à surmonter la crise dans le court et moyen terme, elle l'aggrave. Et ce d'autant plus que les mesures adoptées sont peu cohérentes, y compris du point de vue de l'orthodoxie, de la doxa néolibérale, car elles sont le résultat 
d'intérêts contraires, de concessions et recherche d'une légitimité perdue en raison $1 /$ des conflits entre la Présidence et le ministère de l'économie, tout au moins à l'époque où Lévy, venant de la Bradesco, en était le ministre, $2 /$ de la crise de représentation, les partis se divisant sur la politique à suivre, $3 /$ de la très faible crédibilité du gouvernement. La crise est à la fois une crise de légitimation et une crise de rationalité.

La crise a été systématiquement sousestimée par les instituts de prévision, au point que toutes les deux semaines, les prévisions, révisées, devenaient de plus en plus pessimistes. La chute du PIB est la plus élevée depuis plus de trente ans. Nous l'avons vu. Celle du Pib par tête est encore plus nette (voir Tableau 3). Les perspectives de reprise s'éloignent. II n'est pas certain qu'en 2017 une récupération économique ait lieu... L'appareil industriel se rétrécie a vue d'œil. Le taux d'investissement chute en effet très fortement (Tableau 4). La baisse cumulée sur douze mois de janvier 2015 à janvier 2016 est pour le secteur des biens d capital de $-27 \%$, pour les biens intermédiaires de $-6 \%$ et pour les biens de consommation de $-9,9 \%$, celle affectant les biens de consommation durables étant de $-19,9 \%$ selon l'IBGE. La désindustrialisation se poursuit à un rythme accéléré malgré une très légère reprise au début de 2016 (Figure 4).
Chômage en hausse, revenus réels en baisse sont le reflet d'une crise sociale profonde.

En une année le taux de chômage a doublé et les perspectives d'une aggravation sont de plus en plus crédibles. II devrait passer de $8,4 \%$ de la population économique active à $12 \%$ en 2016 et $13 \%$ en $2017 .$. selon la Pesquisa Nacional por Amostra de Domicílios Contínua (PNADC). Le revenu réel moyen, soit le revenu nominal déflaté du taux d'inflation, connait une chute considérable: $-7,4 \%$ en une année. La création nette d'emplois formels chute, plus particulièrement dans l'industrie de transformation et dans la construction civile et les services, mais le pourcentage des emplois informels croit dans les emplois totaux, après avoir connu une réduction lors des dix années précédant l'avènement de la crise. Selon les enquêtes de la PNAD, l'emploi formel dans le privé baisse passant de $39,2 \%$ à la fin de 2013 à 38,4\% en novembre 2015. La précarité des emplois crées augmente et la flexibilité salariale s'accentue, les augmentations nominales étant en deça du taux d'inflation, ce dernier se situant autour de 10\% fin 2015 (Figure 5) et davantage si on considère l'indice des prix des produits alimentaires, produits ayant une part relative dans le revenu d'autant plus élevé que celui ci est faible.

Avec la hausse du taux d'intérêt nominal Sélic, la part du service de la dette dans le budget

Tableau 3 - PIB ET PIB par habitant, 2000 a 2015

\begin{tabular}{c|c|c|c|c|c|c|c|c|c|c|c|c|c|c|c|c}
\hline & 2000 & 2001 & 2002 & 2003 & 2004 & 2005 & 2006 & 2007 & 2008 & 2009 & 2010 & 2011 & 2012 & 2013 & 2014 & 2015 \\
\hline $\mathrm{IB}$ & 4.4 & 1.4 & 3.1 & 1.1 & 5.8 & 3.2 & 4 & 6.1 & 5.1 & -0.1 & 7.5 & 3.9 & 1.9 & 3 & 0.1 & -3.8 \\
\hline $\mathrm{IB} / \mathrm{t}$ & 2.8 & 0.0 & 1.7 & -0.2 & 4.4 & 2 & 2.8 & 4.9 & 4 & -1.2 & 6.5 & 2.9 & 1 & 2.1 & -0.8 & -4.6 \\
\hline
\end{tabular}

Source: Préparé selon les données BACEN, IBGE.

Tableau 4 - Taux de croissance des investissements

\begin{tabular}{l|l|l|l|l|l}
\hline $3^{\circ}$ trim. 2008 & $3^{\circ}$ trim.2009 & $3^{\circ}$ trim.2010 & $4^{\circ}$ trim.2012 & $1^{\circ}$ trim.2014 & $4^{\circ}$ trim.2015 \\
\hline $14.4 \%$ & $-4.1 \%$ & $19.5 \%$ & 0.8 & 6.1 & -14.1 \\
\hline
\end{tabular}

Source: Préparé selon les données IBGE.

NB: Accumulé sur 4 trimestres en pourcentage, corrigé des variations saisonnières.

Figure 4 - Evolution de la production industrielle, base 2002 = 100, 2002 à janvier 2016

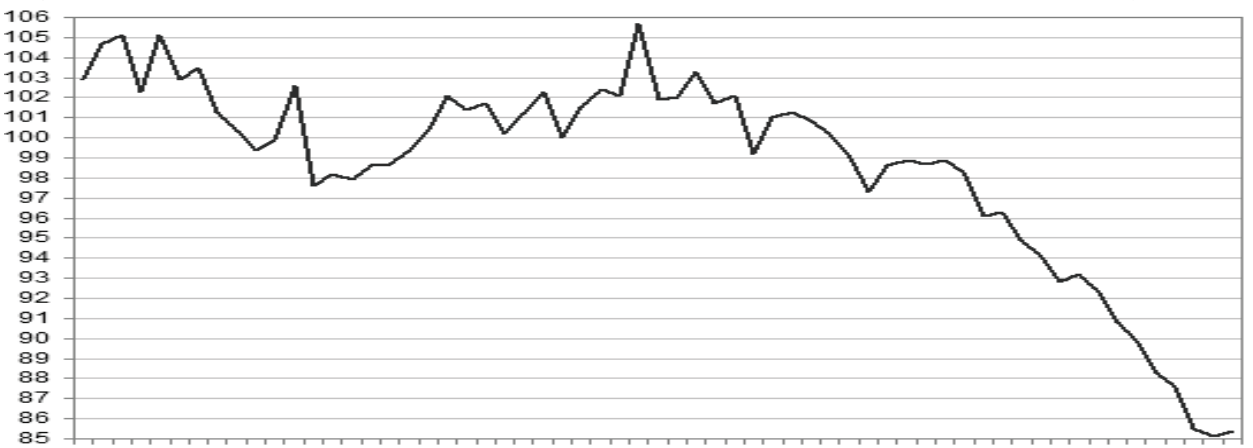

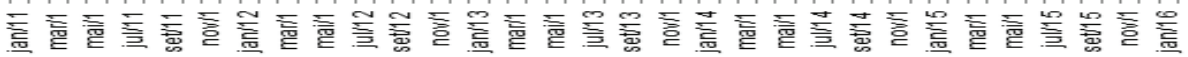

Source: INSTITUTO DE ESTUDOS PARA O DESENVOLVIMENTO INDUSTRIAL. A indústria em janeiro de 2016: por enquanto, avanço pontual. Carta IEDI, São Paulo, n. 722, 2016. Enquête mensuelle, corrigé des variations saisonnières. 
Figure 5 - Taux d'inflation, prix libres et prix administrés

MEDIA DAS EXPECTATIVAS PARA PREÇOS LIVRES E PREÇOS ADMINISTRADOS PARA 2015

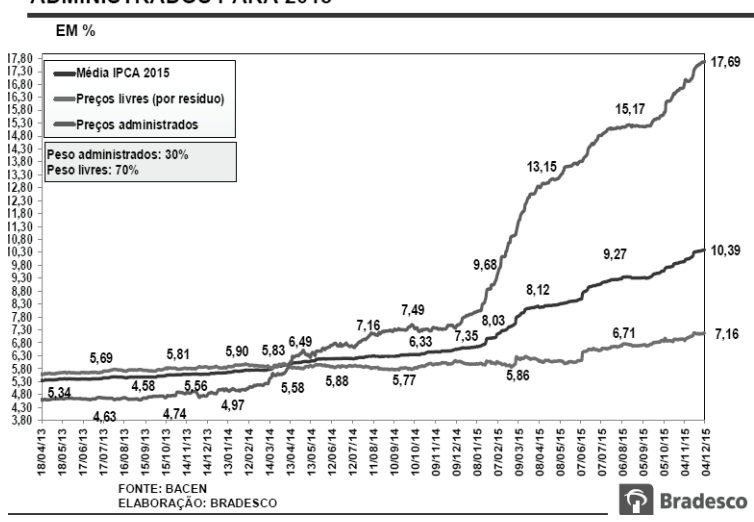

de l'Union croit fortement et se situe dès à présent aux alentours de $45 \%$ des dépenses publiques c'està-dire à un niveau relatif plus important que celui estimé en Octobre 2015 (Figure 6). Celle-ci devrait augmenter d'abord parce que le déficit public croit et qu'il implique un gonflement de la dette, donc de son service, ensuite parce qu'il est possible que les taux d'intérêt réels continuent à augmenter malgré une efficacité contestable sur la hausse des prix, celle-ci s'étant accélérée malgré la hausse passée du taux Sélic, une part importante de celle-ci étant imputable à la hausse très importante des prix des produits dits administrés. Mieux, à l'inverse, on est en droit de considérer que son effet sur l'ampleur des dépenses publiques au titre du service de la dette est de nature à aggraver le déficit budgétaire. Allons un peu plus loi et prêtons-nous à un petit exercice mathématique. Le déficit public se situe aux environs de $10 \%$ du PIB, les recettes publiques, en baisse compte tenu des effets mécaniques de la crise sur les revenus (impôts directs) et la consommation (impôts indirects), se situent aux environs de $30 \%$ du PIB, les dépenses publiques atteignent un peu plus de $40 \%$ du PIB, dont un peu plus des $2 / 5^{\circ}$ sont destinés au service de la dette, soit $16 \%$ du PIB, c'est-à-dire le double de ce qui est consacré au dépenses pour les retraites. Reportons à présent les dépenses au titre du service de la dette par rapport aux recettes. Celle-ci absorbent plus de la moitié des recettes (16/30). II reste donc $14 / 30$ pour payer l'ensemble des dépenses de l'Etat: retraites, éducation, santé, justice, armée et police, transferts sociaux etc. Comme $85 \%$ des dépenses publiques sont à caractères obligatoires - les modalités comme par exemple les mécanismes d'indexation des retraites au salaire minimum sont définies par la loila conclusion est simple: le déficit ne peut qu'enfler démesurément, les dépenses publiques ne peuvent baisser sensiblement, par contre les recettes chutent avec la l'approfondissement de la crise. Le déficit n'a pas d'effet positif sur la croissance puisqu'il est le produit d'une hausse des dépenses au titre du service de la dette, elle-même génératrice d'une augmentation des inégalités, la part des riches dans le revenu augmentant.

Figure 6 - Déc omposition des dépenses publiques de l'Union ${ }^{7}$

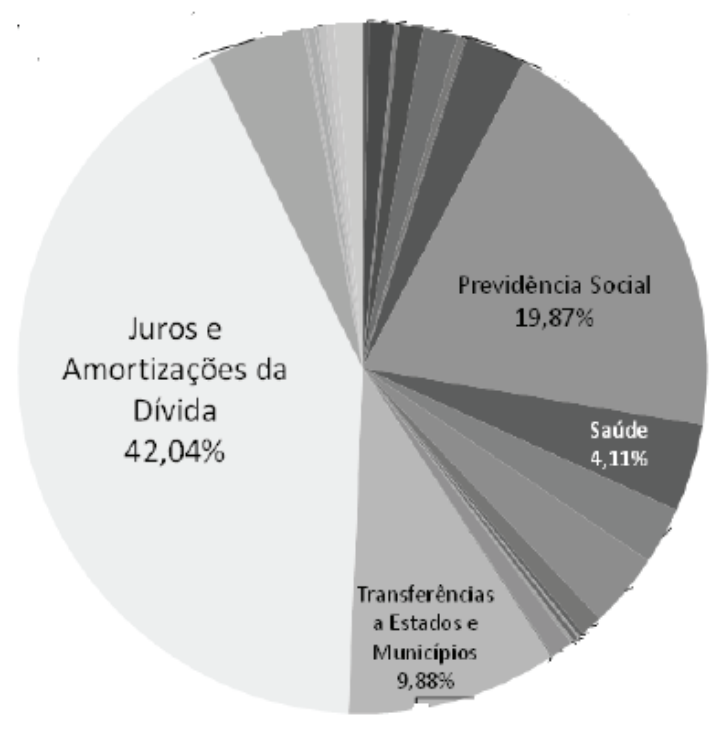

Source: Préparé selon les données Senado Federal - Sistema Siga Brasil. Elaboração: Auditoria Cidadã da Dívida.

La solution pour sortir de la crise parait donc simple: elle a pour nom ajuste fiscal ce qui en terme claires signifie surtout réduction des dépenses publiques ce qui, comme nous l'avons vu, est très difficile à faire à moins de changer la loi. Comme il apparait, dans l'état actuel du rapport de force et de la crise, qu'il ne soit guère possible ni d'envisager une réduction du taux d'intérêt Selic, malgré son inefficacité prouvée, ni d'envisager un gel voire une euthanasie des rentiers préconisée dans son temps par Keynes, fût-elle provisoire, il reste la possibilité de diminuer le poids du second poste du budget, a savoir le retraites (un peu moins de $20 \%$ des dépenses publiques). Sans le paiement des retraites le déficit public disparaitrait... Mais pour cela il faudrait changer profondément la loi et passer d'un système de répartition à un système de capitalisation intégral. Dans l'état actuel du contexte politique, ce n'est pas possible, aussi les mesures envisagées consistent à diminuer la charge budgétaire de ces retraites en préconisant principalement d'allonger la durée totale de la vie active, de chasser les fraudeurs, de diminuer les retraites de reversion et, à terme, de désindexer les retraites du salaire minimum, lequel pourrait luimême être désindexé de l'évolution des prix (et du PIB). Cette dernière mesure une baisse importante des dépenses publiques et une réduction sensible des retraites perçues par le troisième âge, ce qui ne serait pas sans conséquence sur l'augmentation de la pauvreté ${ }^{8}$. Les inégalités augmentant, une reprise 
économique a partir d'un dynamisme retrouvé du marché intérieur ferait défaut et ne parviendrait pas à suppléer le peu de dynamisme du commerce mondial qu'on observe depuis deux années. Le chômage et l'informalité continuerait alors à enfler.

D'autres solutions que celle de l'ajustement fiscal, existent. Les effets délétères de cette politique accentuent et aggravent une crise dont l'origine n'est pas dans la chute du cours des matières premières, même si celle-ci l'a précipitée. C'est dire l'urgence de sortir des sentiers battus (le fameux: there are no alternative de Tatcher) et d'imaginer des solutions nouvelles qui soient en même temps des solutions à la crise. Mais pour les imaginer, encore faut-il ne pas se situer dans le déni et mesurer à la fois l'ampleur de la crise économique et ses causes profondes. Des réformes structurelles ne peuvent s'imposer que si un rapport de force est engagé de manière claire. En ce sens, elles ne sont pas que techniques, elles sont aussi et surtout politiques. Elles concernent 1/ l'instauration d'un système fiscal progressif de nature à dynamiser le marché intérieur sans alourdir pour autant les coûts pour les entreprises, 2/ une nouvelle approche de la politique industrielle ciblée, privilégiant d'abord les industries a fort potentiel de création d'emplois, et celles porteuses d'avenir industriel, 3/ et surtout, à court terme, le vote d'une loi limitant l'emprise croissante du service de la dette interne dans les dépenses budgétaires. Cette loi permettrait d'éviter les effets dévastateurs sur les autres postes de la dépense publique lorsqu'à dépense publique constante celles consacrées au service de la dette interne enflent démesurément, suite aux taux d'intérêt extrêmement élevés.

\section{CONCLUSION}

Cette crise vient de loin. Elle était prévisible. Nous l'avions souligné. Rares étaient les économistes qui l'annonçaient aveuglés par l'aisance financière, la baisse de la pauvreté et le nouvel eldorado. Elle se paie aujourd'hui d'autant plus fortement que l'appareil industriel s'est détérioré avec le développement des comportements rentiers (reprimarisation de l'économie, financiarisation). Aujourd'hui, avec les taux d'intérêt extrêmement élevés, seules les couches liées à la finance ne souffrent pas de la crise, voire en bénéficient. Etranglé par les contraintes budgétaires, le choix devient de plus en plus simple: soit le gouvernement poursuit sa politique de contention des dépenses publiques et accroit la crise et la désespérance sociale, soit il coupe dans les dépenses liées au service de la dette interne et décident de limiter ce service à 25 ou $30 \%$ de l'ensemble des dépenses, dégageant ainsi des possibilités de maintenir des dépenses sociales (retraite, santé, éducation quitte à favoriser des réformes), de favoriser les dépenses de recherche, d'impulser de vraies politiques industrielles rompant avec celles menées par le passé. Limiter les dépenses affectées au service de la dette, via une baisse des taux d'intérêt anormalement élevés, n'est pas plus révolutionnaire que limiter les dépenses publiques lorsque les recettes publiques baissent. C'est ce qui fut décidé sous la présidence Reagan aux Etats Unis avec la loi Gramm-Rudman-Holings en 1985.

La crise est politique. La corruption qui mine l'ensemble des partis est aujourd'hui refusée par la base, la population. C'est une observation très importante qu'on peut faire pour presque tous les pays latino-américains. La corruption n'est plus considérée comme une fatalité, voire un accommodement avec les législations trop lourdes, la multiplicité des partis et la difficulté de construire des coalitions durables pour gouverner. C'est un fait positif. La corruption est d'autant plus rejetée que la crise économique est présente, qu'elle a un coût social de plus en plus insupportable. Mais elle est aussi instrumentalisée par l'opposition qui, recevant l'appui des principaux moyens de communication, ne rêve que d'une chose: marginaliser le PT et revenir au pouvoir. De ce point de vue, elle s'apparente à une tentative de coup d'Etat médiatico-juridique. Défendre aujourd'hui les hommes et femmes politiques du PT c'est dans une certaine mesure tomber dans le piège de défendre des corrompus ou supposés tels et ce à un moment où le PT paie au prix fort la politique d'austérité menée depuis le second mandat de Dilma. Aussi vaut il mieux se concentrer sur la défense des intérêts économiques (salaire, retraite, indexation, limitation du service de la dette...) tout en dénonçant les manœuvres politiciennes $d$ e l'opposition et sa pratique également corruptive.

La logique économique n'est pas souvent compatible avec la logique politique. La crise politique que connait la présidence de Dilma Rousseff rend difficile d'imaginer des politiques alternatives, sauf si elle trouve un dépassement par des mobilisations populaires. La crise politique aggrave la crise économique, et vice et versa, pouvant entrainer une crise institutionnelle. On le voit l'enjeu n'est pas qu'économique. II est aussi politique.

\section{RÉFÉRENCE ${ }^{9}$}

SALAMA, P. Argentina, Brasil, México entran en la tormenta. ¿Quo vadis América Latina? Herramienta: debate y critica marxista, Buenos Aires, 2015. Publié en français dans le site de la FMSH comme working paper. Disponible :<http://www.herramienta.com. ar/herramienta-web-17/argentina-brasil-mexicoentran-en-la-tormenta-quo-vadis-america-latina>. Accés: 1 jan. 2016.

\section{NOTES}

1 Encore faut-il rappeler que dans les pays du Sud, la 
pauvreté est mesurée de manière absolue. II est donc possible de l'éliminer. A l'inverse, dans les pays dits avancés, elle est mesurée de manière relative. On ne peut donc l'éliminer. Elle peut diminuer ou bien s'accroitre. Elle s'est d'ailleurs accrue avec l'essor des inégalités et la tendance à la stagnation économique.

2 La très forte dépréciation du Real par rapport au dollar, entre $40 \%$ et $50 \%$, rend les importations de plus en plus coûteuses. Celles-ci baissent fortement $(-13,9 \%$ en 2015) sous l'effet conjugué de la dépréciation de la monnaie et de la baisse de la production et de la consommation des brésiliens. Dans un premier temps, la balance commerciale dégage un solde de plus en plus positif grâce à une baisse plus élevée des importations que des exportations, dans un second temps, la baisse des importations se poursuit, mais les exportations de produits manufacturés de février 2015 à février $2016: 7,9 \%$ et semi manufacturés: 14\% sont dopées par la dépréciation de la monnaie - malgré un contexte global de ralentissement du commerce mondial - et le solde de la balance commercial devient davantage positif malgré la baisse des exportations de matières premières: $-0,5 \%$ (données de Secex/ Mdic). Parallèlement, les sorties de capitaux au titre du paiement des dividendes ralentissent, non seulement parce que les dividendes versés sont plus faibles mais aussi parce que les expatrier avec un taux de change qui se déprécie est moins avantageux que de le les conserver pour partie au Brésil en attendant des jours meilleurs en termes de taux de change ; il en de même pour le tourisme a l'étranger le taux de change rendant plus couteux des voyages à l'étranger. II en résulte que le solde de la balance ces comptes courants (voir supra définition en note) est moins négatif que par le passé et les besoins de financement, jusque là comblés par les entrées de capitaux au titre surtout des investissements étrangers directs et des investissements en portefeuille, moins forts. La contrainte externe est moins élevée.

3 L'indice des prix a la consommation (IPCA) croit de $10,67 \%$ en 2015 et l'indice des prix correspondant aux paniers de consommation des ménages aux revenus modestes (INPC) augmente de $11,28 \%$.

4 Le coût unitaire du travail est un premier indicateur de la compétitivité. Trois variables le définissent: le salaire réel, la productivité et le taux de change.

5 La reprimarisation de l'économie est la conséquence directe de la hausse des cours des matières premières et de la hausse des volumes vendus surtout à la Chine, celle-ci devenant le premier partenaire commercial du Brésil. La contrainte externe disparait alors d'autant plus qu'augmentent également les entrées de capitaux. Ces entrées consécutives de devises permettent une augmentation des réserves internationales et surtout conduisent à une appréciation forte de la monnaie nationale. Celle-ci provoque déformation des prix relatifs, les importations devenant relativement moins chères et, compte tenu du contexte de libéralisation des échanges, le taux de rentabilité des entreprises a tendance a baisser, ce qui provoque une désindustrialisation dite précoce, connue sous le nom de maladie hollandaise. Lorsqu'elle n'est pas contrée par une politique active sur les taux de change, visant à limiter, voire annuler l'appréciation de la monnaie nationale, la maladie hollandaise accentue le processus de désindustrialisation.

6 Sur cette question voir Salama (2012, 2014 apud
SALAMA, 2015) et la bibliographie citée dans ces livres et articles

7 En général, on considère que les intérêts sont payés par l'impôt et l'amortissement de la dette par l'émission de bons du Trésor. C'est ce qui explique que très souvent on n'inscrit dans les dépenses publiques que le service de la dette du au paiement des intérêts. Ce n'est pas le cas dans ce graphique. Aujourd'hui, l'amortissement dépasse en valeur le paiement des intérêts $(8.5 \%$ du PIB). Cette convention comptable est contestable car le déficit budgétaire croissant est financé par des émissions de bons, or ce déficit résulte pour une part importante de la hausse du taux d'intérêt.

8 Rappelons que selon le Ministerio da Previdencia, $66,7 \%$ des bénéficiaires ont un salaire minimum, $15 \%$ entre 1 et $2,7,9 \%$ entre 2 et $3,5,1 \%$ entre 3 et $4,2,3 \%$ entre 4 et 5 et enfin $0.6 \%$ plus de 5 salaires minimums.

9 Voir les références aux données statistiques dans le texte. Nous nous limiterons ici à un article récent où on pourra trouver une bibliographie abondante.

\section{Pierre Salama}

Economista

Doctorat d'Etat Paris Sorbonne

Professeur émérite des Universités Centre d'Économie de Universidade Paris XIII

E-mail: pierre.salama@univ-paris13.fr

\section{Universidade Paris XIII}

Université Paris 13 - Campus Villetaneuse

99 avenue Jean-baptiste Clément

93430 Villetaneuse 